\title{
THE IMPACT OF RAIN, FROST, SEASONAL CYCLE, AND WIND ON SEQUENCES OF HIGH RESOLUTION URBAN SAR IMAGES
}

\author{
Gottfried Schwarz and Mihai Datcu
}

\author{
German Aerospace Center (DLR), Remote Sensing Technology Institute, Oberpfaffenhofen, Germany
}

\begin{abstract}
The TerraSAR-X satellite has been fully operational since nearly 6 years and has delivered a very large quantity of high resolution SAR images. Among these images one can find a number of repeated acquisitions of selected target areas taken with nearly identical imaging parameters. These image time series data often cover the full seasonal cycle of a target area and lend themselves well to small-scale change detection; however, in the case of urban areas, we have to be aware of the quantitative impact of rain, frost and wind on high resolution SAR images. In particular, images of Western European cities are characterized by construction work concentrating on single buildings within a fully built-up city and by public green space changes. A quantitative analysis of urban time series data has to discriminate between definite changes of the urban landscape and the transient impacts of rain, frost and wind. The critical issue is how to identify and characterize these transient phenomena.
\end{abstract}

Index Terms - SAR imaging, image time series, urban areas, TerraSAR-X, rain, frost, wind

\section{INTRODUCTION}

When we want to analyze changes in high resolution SAR image time series and to retrieve specific image content, we have to make sure what target features we want to study, and what image acquisition and processing effects have to be calibrated and corrected for prior to image analysis. If we concentrate on morphological changes in images of urban areas such as new buildings, road construction work, or the demolition of industrial facilities then we are mostly interested in the actual shape of human made objects. In these cases, we are less interested in changes solely due to differences in image acquisition or processing parameters of single images, or isolated atmospheric effects. As a consequence, we have to be sure how to discriminate various effects due to image geometry and image radiometry.

\section{SAR IMAGE GEOMETRY AND RADIOMETRY}

As for the geometry of SAR image time series, we do not only have to make sure that all images of a series cover the same overlap area on ground but, ideally, all images should be taken under the same viewing conditions and should have the same projection, scale, resolution, and occlusions. This can be accomplished by relying on observations with identical recording parameters taken from a recurring position along a repeat orbit and by selecting the same image processing options. In the case of TerraSAR-X products, we have very good internal geometric image stability that even allows for a comparison of geometrically un-rectified images after some final horizontal and vertical alignment (with or without sub-pixel accuracy). Thus, we can select candidate images that comply with the repeat orbit constraint and have identical processing parameters [1]. We only have to find the common overlap area of all images and have to co-align and stack them.

As for the radiometry of SAR images, many time series analysis algorithms rely on images that are properly calibrated and thus become inter-comparable in brightness. This may include, for instance, the removal of a thermal noise offset. However, we also know that a number of often unaccounted effects resulting from rain or soil moisture, frost, snow or ice cover, the seasonal cycle of vegetation, or wind may have a considerable impact on the actual brightness and the contrast of a given SAR image [2,3,4, and 5]. In the following, we discuss which of these effects can be identified and corrected for during our image analysis steps, and what consequences we have to bear with uncorrected images when it comes to change detection within time series data.

\section{SELECTED TEST DATA SET}

In order to quantify the radiometric impact of the various potential effects listed above, we selected a time series of 22 overlapping high resolution SAR images taken with the TerraSAR-X instrument over the city of Berlin, Germany (cf. Table 1 and Fig. 1). These images were acquired from a recurring position of the 11 day TerraSAR-X repeat orbit at the same local time. We selected this data set as it has no interruptions of the sampling intervals and allows us to monitor the seasonal cycle on a gap-free regular time grid of 11 days. Thus, we circumvent any potential interpolation effects that might degrade the analysis of temporal evolutions.

Each image does not only cover fully built-up areas but also includes, for instance, public parks and waterways. 
Therefore, we split the images into sub-scenes with main target classes (built-up areas with big administration buildings, built-up areas with medium-sized residential buildings, industry and harbor areas, public parks, and water bodies).

\begin{tabular}{|c|c|}
\hline Instrument & TerraSAR-X \\
\hline Recording mode & High res. spotlight mode \\
\hline Polarization & VV \\
\hline Orbit branch & Descending \\
\hline Acquisition time & 05:17 UT \\
\hline Product level & $\begin{array}{l}\text { MGD: Multi Look Ground } \\
\text { Range Detected }\end{array}$ \\
\hline Product option & $\begin{array}{l}\text { Radiometrically enhanced } \\
\text { (i.e., noise corrected, etc.) }\end{array}$ \\
\hline $\begin{array}{l}\text { Center of target area } \\
\text { (lat. / lon.) }\end{array}$ & $52.5 \mathrm{~N}, 13.4 \mathrm{E}$ \\
\hline Incidence angle & $46.6^{\circ}$ \\
\hline Columns / lines per image & са. $5700 / 4300$ \\
\hline Image resolution & $2.86 \mathrm{~m}$ \\
\hline Pixel spacing & $1.25 \mathrm{~m}$ \\
\hline Nominal signal-to-noise & $25.02 \ldots 26.46$ \\
\hline $\begin{array}{l}\text { Acquisition dates } \\
\text { (dd_mm_yyyy) }\end{array}$ & 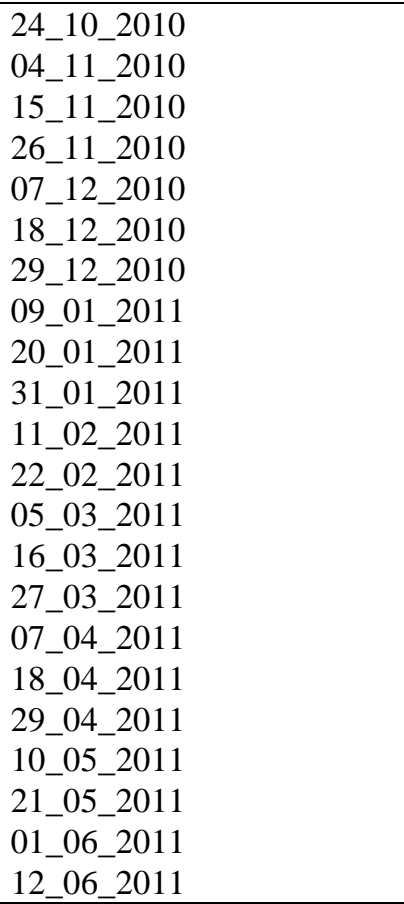 \\
\hline Wind measurement station & $\begin{array}{l}\text { Berlin-Pichelsdorf, Ger- } \\
\text { many }\end{array}$ \\
\hline
\end{tabular}

Table 1: Image test data set

The analysis of the selected sub-scenes allowed us to identify and quantify the effects of surface moisture, frost, vegetation cycle and wind for each primary target class. For all image acquisition dates we also collected meteorological data provided via Internet by the Institute of Meteorology of the Freie Universität Berlin as well as from the archives of the public website www.wetterspiegel.de

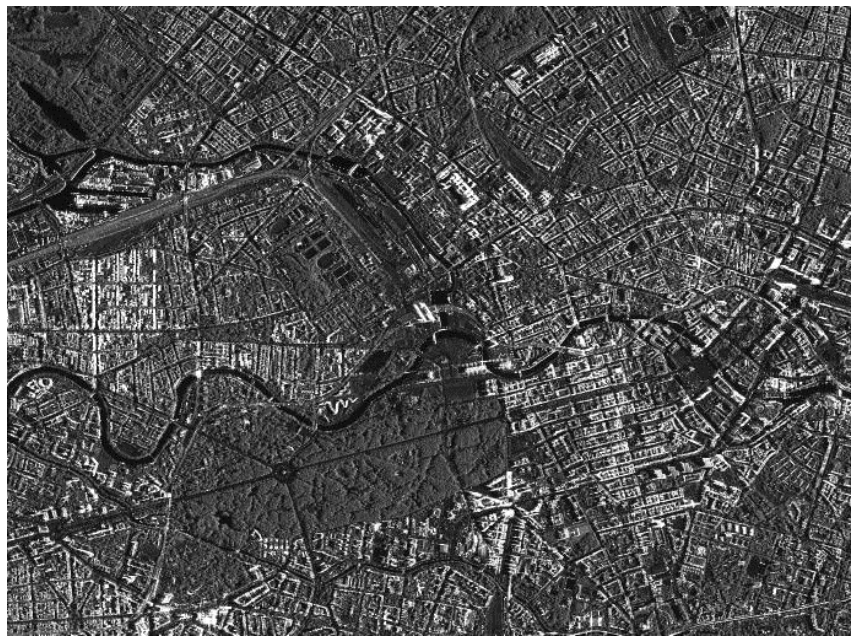

Fig. 1: TerraSAR-X scene of Berlin, Germany

\section{RAIN AND FROST EFFECTS IN BUILT-UP AREAS}

The effects of strong rainfall signatures recorded during SAR imaging are described in detail by [4] and [5] and do not need to be elaborated further in this publication (as there are not so many strong early morning rainfall events in central Europe). However, in the more frequent case of increased SAR backscattering due to elevated soil or surface moisture resulting from precipitation accumulated over several hours, one can easily demonstrate the magnitude of the phenomenon, for instance, via the mean value of each image or via the de-trended singular values of each image [6]. This holds for our test data set as the images are highly similar, and the main changes from image to image are limited to construction work of single buildings or vegetation cycle effects within small local patches of an extended urban area.

The singular value decomposition leads to easily understandable results. In most cases, a plot of the de-trended singular values of our images (taken over built-up urban areas) shows rather similar values. The only exceptions are moist surface images which result in noticeably larger values, and images taken during extended frost periods with reduced backscattering. This is illustrated in Fig. 2 that shows the over-plotted and de-trended (i.e., mean profile subtracted) singular values of 22 overlapping sub-scenes of a built-up area in Berlin, Germany. One can easily discriminate three cases with enhanced backscatter and three other cases with reduced backscatter.

The validity of these results was verified by an analysis of the aforementioned meteorological data and by an additional comparison with image annotation data. Moist conditions are typically accompanied by higher backscatter and low nominal signal-to-noise levels becoming apparent during 
routine product generation and will be recorded as image metadata annotations, while frost periods with dry soil and snow result in lower backscatter and higher nominal signalto-noise ratios. It has to be noted, however, that the meteorological data are only given for time intervals of several hours and, in most cases, the measurement stations are located at least some kilometers away from a selected sub-scene of an image. As a consequence, we cannot be absolutely sure about the true surface moisture at a given location or the actual wind conditions prevailing there during the few seconds of image acquisition.

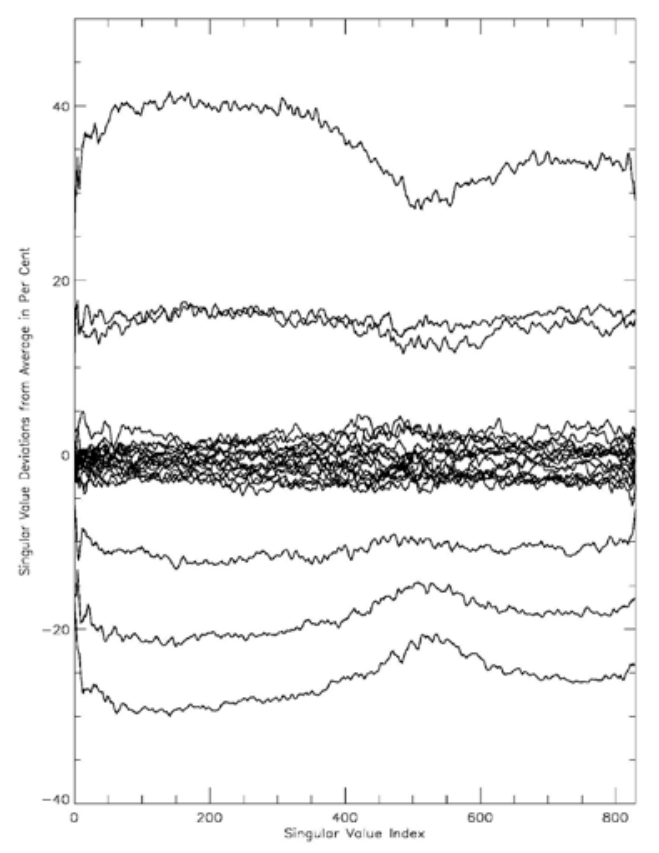

Fig. 2: Soil moisture and frost seen by singular values

Once we have identified moisture or frost effects, we have to make sure how the increased or decreased brightness levels will affect the analysis of time series data. If our algorithms are susceptible to brightness variations then we have several options to select from:

- If we have a sufficient number of good images and if we can tolerate data gaps then we can simply leave out the most offending images

- If we dispose of a robust correction technique we can correct the offending images. A typical approach is a correction formula such as $\mathrm{Y}_{\mathrm{i}}=\mathrm{a}+\mathrm{bX} \mathrm{X}_{\mathrm{i}}$

- Experienced user can work with band difference ratios of image pairs $\left(\mathrm{X}_{\mathrm{i}}-\mathrm{X}_{\mathrm{j}}\right) /\left(\mathrm{X}_{\mathrm{i}}+\mathrm{X}_{\mathrm{j}}\right)$ to compensate brightness level discrepancies. This technique can be applied per pixel to account for distinct surface cover types with different moisture responses.

\section{FROST EFFECTS ON WATER BODIES}

The singular value technique also shows peculiar frost effects for water bodies. In the case of permanent frost, ice developing on the water surfaces results in greater singular values. In contrast, during warmer weather, or in case of rainfall prior to image acquisition, the singular values are not affected. However, for our applications, we see no need for a correction of the ice effects as the extent of ice cover can be regarded as a change analysis phenomenon to be investigated in detail.

\section{SEASONAL CYCLE AND WIND EFFECTS}

A public park with deciduous trees will show a seasonal behavior. While the radar backscatter during wintertime mainly depends on the soil characteristics, a dense canopy during summertime will have its own X-band backscatter characteristics; during the transition periods, mixed backscattering signals will be recorded. Thus, we have a much broader distribution of responses than in the case of built-up areas.

In order to identify and to discriminate the various vegetation stages and the effects of moisture, one can resort to pixel brightness histograms. The local histogram shapes of our 22 public park sub-scenes differ markedly and a further complication arises due to wind resulting in tree branches and twigs moving during image acquisition $[7,8,9]$. In a SAR image, a target object moving along flight direction will be smeared, and this leads to a loss of contrast; edges and radar shadow areas will disappear.

Therefore, we can try to decompose brightness histograms of public park sub-scenes into their components. Figure 3 shows three typical cases with different component contributions. Instead of the usual gamma distributions of urban SAR image histograms [10], we can see a simple monomodal soil brightness histogram in winter, which gets transformed into a bi-modal soil-plus-canopy histogram in springtime and summer (with a maximum canopy contribution in late summer); thus, soil and canopy contributions will vary in their percentages. Interestingly, the canopy may be darker or brighter than the soil; a cause may be the longer lifetime of soil moisture variations compared to the backscattering effects of tree leaves.

We also have to consider wind which will smear out the shadows between tree crowns and thus creates a shift of the "dark edge" of the brightness histogram towards a monomodal curve. According to our experience, we could definitively identify wind with a speed of about $8 \mathrm{~m} / \mathrm{s}$ in tree canopies. It has to be noted, however, that our comparisons rely on nominal local wind gust speeds as published by local meteorological institutions.

Things become rather involved when we try to correct wind effects in vegetation scenes. Users performing change detection will aim at the undisturbed seasonal cycle of vege- 
tation. Therefore, we have to preserve the radiometric peculiarities of the basic vegetation processes and one could think of a correction of moisture induced and wind related effects. At present, however, we are not aware of an efficient wind related correction algorithm and we can only suggest to analyze all histograms in detail and to discard all offending data sets before one carries out blind change detection.

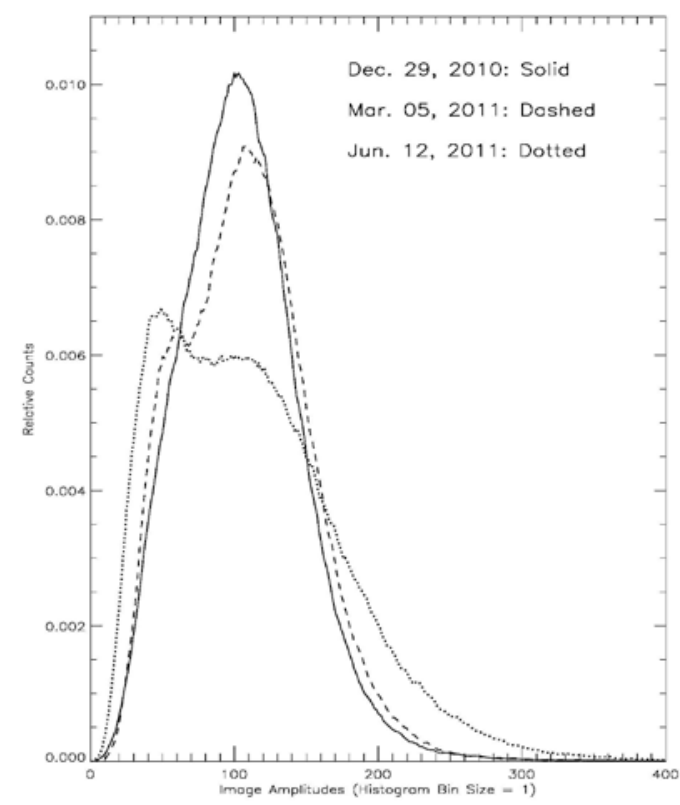

Figure 3: Brightness histograms of a public park scene

\section{WATER SURFACE EFFECTS}

Wind does not only affect the imaging of vegetation. Water bodies represent another surface type that is very susceptible to the prevailing wind [11]. When we concentrate on water surfaces in urban areas we are mainly concerned with small lakes, rivers, and water ponds; in contrast, we are less interested in ocean surface phenomena. Thus, a typical topic for the analysis of urban SAR images is the monitoring of the intactness of bridges crossing a river, for instance, after an earthquake.

When we set out to analyze bridges in satellite SAR images, we can profit from single, double and triple reflections. Single reflections tell us about the deck of a bridge, double reflections characterize the lateral bridge details that reflect back to the water surface and then to the SAR satellite, while triple reflections (satellite - water surface - lower side of the bridge - water surface - satellite) characterize the width of the bridge seen "from below" $[12,13]$. Here the water surface acts as a reflector for radar signals. The wind dependent smoothness of the water surface is a critical parameter.

\section{REFERENCES}

[1] H. Breit and 5 co-authors, "TerraSAR-X Payload Data Processing: Results from Commissioning and Early Operational Phase”, Proc. IGARSS 2008, pp. II209-II212, Boston, USA

[2] C.J. Oliver and S. Quegan, "Understanding synthetic aperture radar images”, Artech House, 1998

[3] T. Schellenberger, B. Ventura, M. Zebisch, and C. Notarincola, "Wet Snow Cover Mapping Algorithm Based on Multitemporal COSMO-SkyMed X-Band SAR Images“, IEEE Journal of Selected Topics in Applied Earth Observations and Remote Sensing, Vol. 5, pp. 1045-1053, 2012

[4] A. Danklmeier, M. Chandra, "Precipitation Induced Signatures in SAR Data”, Proc. EuCAP 2009, pp. 3433-3437, Berlin, Germany

[5] F.S. Marzano, S. Mori, and J.A. Weinman, "Evidence of Rainfall signatures on X-Band Synthetic Aperture Radar Imagery Over Land”, IEEE Trans. on Geoscience and Remote Sensing, Vol. 48, pp. 950-964, 2010

[6] http://en.wikipedia.org/wiki/Singular_value_decomposition

[7] S.U. Pillai, K.Y. Li and B. Himed, "Effect of Wind on SpaceBased Radar Performance”, IEEE Radar Conference 2008, pp. 15, Rome, Italy

[8] H. Chen, Y. Sun, Y. Chen, J. Cao, “A Fast Simulation Method for Dynamic Forest in Wind", Proc. Cross Strait Quad-Regional Radio Science and Wireless Technology Conference 2011, Vol. 2, pp. 1339-1342, Harbin, China

[9] M. Benson, L. Pierce, K. Sarabandi, “Quantifying the results of wind and rain on IFSAR tree height estimation", Proc. IGARSS 2010, pp. 3275-3278, Honolulu, Hawaii

[10] H.-C. Li, W. Hong, Y.-R Wu, and P.-Z. Fan, "On the Empirical-Statistical Modeling of SAR Images with Generalized Gamma Distribution”, IEEE Journal of Selected Topics in Signal Processing, Vol. 5, pp. 386-397, 2011

[11] A.A. Mouche and 7 co-authors, "On the Use of Doppler Shift for Sea Surface Wind Retrieval From SAR”, IEEE Trans. on Geoscience and Remote Sensing, Vol. 50, pp. 2901-2909, 2012

[12] U. Soergel, E. Cadario, H. Gross, A. Thiele, U. Thoennessen, "Bridge Detection in Multi-Aspect High-Resolution Interferometric SAR Data”, Proc. Eusar 2006, Dresden, Germany

[13] U. Soergel, E. Cadario, A. Thiele, U. Thoennessen, "Feature Extraction and Visualization of Bridges over Water from highresolution InSAR Data and one Orthophoto", IEEE Journal of Selected Topics in Applied Earth Observations and Remote Sensing, Vol. 1, pp. 147-153, 2008 